\title{
Biceps Brachii Muscle with Third Head a Case Study
}

\author{
$\begin{array}{llll}\text { S. Bansal } & \text { V. Budhiraja } & \text { S. Swami } & \text { R. Gupta }\end{array} \quad$ N. Gaur ${ }^{1}$ \\ ${ }^{1}$ Department of Anatomy Kalpana Chawla Government Medical \\ College, Karnal, Haryana, India \\ Address for correspondence S. Bansal, Department of Anatomy \\ Kalpana Chawla Government Medical College, Karnal-132001, \\ Haryana, India (e-mail: bansal.swati64@gmail.com).
}

J Morphol Sci 2018;35:14-16.

\begin{abstract}
Introduction Biceps brachii muscle belongs to the flexor group of muscles in arm. As Biceps brachii is double headed muscle. It is one of the known variable muscles of human body in terms of number and morphology.

Material and Method During routine human cadaveric dissection for the purpose of teaching medical undergraduates in our department. The upper limb of embalmed adult male cadaver was dissected as per the standard method to note the origin, insertion, and nerve supply of biceps brachii.

Results The three headed biceps brachii was found unilaterally on right side in adult male cadaver. The long and short head had normal origin, but third head originated from humerus below insertion of coracobrachialis and inserted with main muscle belly

Keywords

- biceps brachii

- number

- third head

- variation and innervated by musculocutaneus nerve.

Conclusion Variant biceps brachii may confuse a surgeon who performs procedures on the arm and may lead to iatrogenic injuries. The surgeons and traumatologists have to keep such muscular variations in mind. So the knowledge of existence of its variation is important for anaesthetists and surgeons.
\end{abstract}

\section{Introduction}

The biceps brachii as the name suggests, biceps mean any skeletal muscle having two heads and brachii means arm. There is a biceps muscle in both forelimbs and hindlimbs i.e., biceps femoris. Biceps brachii muscle belongs to the flexor group of muscles in arm. It is also called biceps flexor cubiti.

It is the only flexor muscle of the arm which crosses the shoulder as well as the elbow joint. As Biceps brachii muscle has two heads, short head and long head. Long head originates from the supraglenoid tubercle of scapula and it is both intraarticular and extrasynovial and short head originates from the tip of coracoid process of scapula. Distally, these two heads join to form a common tendon which inserts on the posterior rough part of radial tuberosity, and some aponeurotic fibers form the bicipital aponeurosis which merges with deep fascia of forearm, this arrangement serves to protect the brachial artery and median nerve during phlebotomy of median cubital vein.

received

April 1, 2017

accepted

February 13, 2018 10.1055/s-0038-1660492. ISSN 2177-0298.
The Biceps brachii muscle is innervated by the musculocutaneous nerve (root values: anterior division of ventral primary rami of $\mathrm{C} 5, \mathrm{C} 6$ ) and supplied by brachial and anterior circumflex humeral arteries. This biceps brachii is a biarticular muscle which means that it helps to control the motion at two different joints shoulder and elbow. The biceps brachii acts as true prime supination of forearm assisted by supinator muscle and act as a synergist to the true prime flexor the brachialis muscle at elbow joint. The long head acts as humeral head depressor and glenohumeral joint stability. The short head aids in adduction of humerus, also act as fixator to stabilize shoulder joint.

\section{Case History}

During the routine undergraduate cadaveric dissection performed in our department of upper limb a three headed biceps brachii was found unilaterally in the right arm of 45-55-year-old adult male cadaver. The human

Copyright @ 2018 by Thieme Revinter

Publicações Ltda, Rio de Janeiro, Brazil

License terms

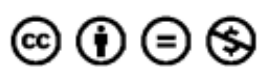


cadaver used in the dissection was obtained through our departmental body donation program following all ethical guidelines.

The arm was dissected carefully to expose the full length of Biceps muscle from proximal to distal attachment. All other related structures were also exposed. The additional head was examined for the origin and course up to its insertion and then photographs were taken (-Fig. 1).

The third head was a flat muscular belly which originated from the shaft of humerus near the insertion of coracobrachialis. Third head was present deep to other two heads. It lies lateral to the brachial artery and median nerve. Third head was supplied by a twig from musculocutaneus nerve. Other two heads of Biceps were supplied by musculocutaneous nerve. Third head descended and merged with other two heads to form common tendon and was inserted on posterior part of radial tuberosity. No other variations were observed.

\section{Discussion}

Variations are inherent in all the species and is necessary to ensure diversity within the species. Biceps brachii is one of the most variable muscles of the human body Occurrence of this variation is population specific with a rate ranging from $2 \%$ (Nayak et al., 2006) ( $^{1}$ to $37 \%^{2}$ of different ethnic groups, out of which Indian population had $2 \%$ variation and highest in Columbians i.e., $37 \%$. It is one of the most variable muscle in human being, third head being a common variation, though supernumerary heads up to 8 have been recorded in previous literature. ${ }^{3}$ Most of the articles mention supernumerary head of biceps occurs unilaterally, and bilateral occurrence is rare. Supernumerary heads are found more commonly in males. ${ }^{4}$
After searching the literature, we came to know extra heads may present as a belly similar to usual heads or may be present as a group of accessory fascicles arising from either of these: - Head or shaft of humerus, coracoid process, pectoralis major or minor tendon, capsule of the shoulder joint or V-Shaped insertion of deltoid muscle. The commonest origin is from the proximal part of humerus as in our case, hence also called as humeral head. Below are some of the researches on third head with different origins.

Kumar et al. ${ }^{5}$ stated that they found bilateral third head of biceps brachii in one male cadaver out of 48 cadavers. Its origin was from the anterior limb of $\mathrm{V}$ shaped deltoid insertion on the humerus and it fused with the common bulk of the muscle before insertion.

Donmez et al. ${ }^{6}$ found supernumerary heads in two male cadavers. In one case the origin was from the medial lip of the intertubercular groove and in another case it was from the lateral lip of intertubercular groove. In both the cases, the three heads united with one another at the middle third of the arm and formed a common tendon before the insertion. Balasubramanian $^{7}$ found supernumerary head in one old adult male cadaver originated from upper and medial part of brachialis and fused with the common muscle belly for insertion.

Singh et al. ${ }^{8}$ observed additional head in both the arms arose from humeral shaft between the insertion of coracobrachialis muscle above and origin of brachialis below in adult male cadaver bilaterally.

Govindarajan and Vellaichamy ${ }^{9}$ found third head arising from capsule of shoulder joint and fused with main muscle in middle of right arm in adult male cadaver.

Shashikala and Ashwini ${ }^{10}$ conducted a study on ten cadavers from which third head was found only in one case originated from $\mathrm{V}$ shape insertion of deltoid muscle unilaterally and inserted with main muscle.

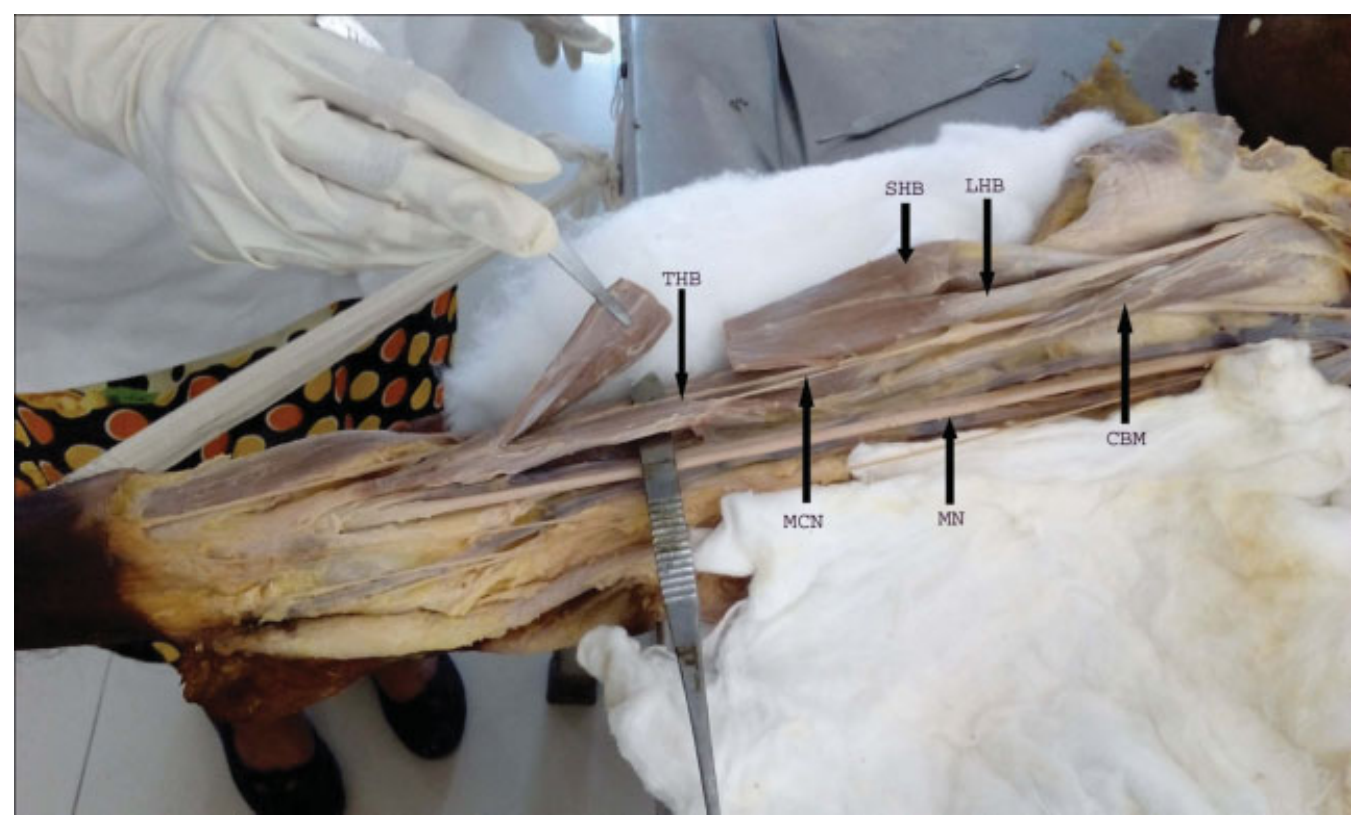

Fig. 1 Third head of biceps brachii arising from humerus near insertion of coracobrachialis (THB-third head of biceps, SHB-short head of biceps, LHB-long head of biceps, CBM-coracobrachialis muscle, MCN - musculocutaneus nerve, MN-median nerve). 
Lokanadhan and Devi ${ }^{11}$ found supernumerary head bilaterally in two cadavers out of 40 cadavers. In one cadaver the third head originated from lower anterior part of humerus on its medial side and fused with common bulk of muscle before its termination into bicipital aponeurosis. In another male cadaver it had its origin from upper anterior part of humerus on its medial side. The third head fused with common bulk of muscle before the origin of bicipital tendon.

Cheema and Singla ${ }^{12}$ found three unilateral supernumerary heads in biceps brachii muscle out of 63 adult cadavers in North Indian Population. Two of the supernumery heads had their origin from the shaft of the humerus near the insertion of coracobrachialis and one had its origin near the origin of brachialis. Their insertion was into the muscle belly in two cases and into the bicipital aponeurosis in one. The supernumery heads were innervated by a branch from musculocutaneous nerve.

As in our case third head is arising from humeral shaft also called humeral head which is most common location of supernumerary head and is supplied by musculocutaneus nerve and inserted into main muscle belly before insertion which is in agreement with the previous reports. ${ }^{8,12}$ The presence of third head of biceps brachii has its functional and clinical implications. From functional point of view third head increases the strength of biceps tendon in elbow flexion and pronation of forearm irrespective of shoulder position. From clinical point of view third head can cause unusual bone displacement subsequent to fracture humerus. If supernumerary head is present unilaterally it can confuse the surgeon for any soft tissue tumor during operation. Variant origin of third head may lead to intramuscular course of musculocutaneus nerve and can cause nerve compression during contraction of biceps especially important in cases of professional body builders and weight lifters where the biceps become hypertrophied due to vigorous exercise. This additional head of biceps can be used as a flap in plastic surgeries. the nerve or the vascular structure is piercing the accessory head, then it would probably cause difficulty during elevating or transferring the flaps.

Explanation for the third head from different views: Evolutionary view- Phylogenetically third head of biceps brachii represent long head of coracobrachoialis, particularly in those cases where the third head arose from the insertion area of coracobrachialis, as in our study also. ${ }^{13}$ Embryological view-Probably the third head of biceps brachii is a portion of brachialis muscle whose insertion is shifted from ulna to radius and innervated by musculocutaneus nerve. ${ }^{2}$ Circula- tory view-During the time of formation of brachial plexus because of presence of many circulatory factors the third head of biceps brachii is present. ${ }^{14}$

\section{Conclusion}

Presence of additional head and its nerve supply is important for clinicians and anaesthetist for selective nerve blocks and to treat the nerve impairments.

\section{References}

1 Nayak SR, Prabhu LV, Sivanandan R. Third head of biceps brachii: A rare occurrence in the Indian population. Ann Anat 2006;188 (02):159-161 Doi: 10.1016/j.aanat.2005.10.004

2 Rincon F, Rodríguez ZI, Sánchez A, Leon A, González LF. The anatomic characteristics of the third head of biceps brachii muscle in a Colombian population. Rev Chil Anat 2001;20(02):197-200

3 Hollinshead WH. Anatomy for surgeons. New York: Hoeber-Harper; 1958

4 Vijayabhaskar P, Baral P, Vaishya R, Shrestha RN. Supernumerary head of Biceps brachii: a rare occurrence in the Nepalese population. Kathmandu Univ Med J (KUMJ) 2008;6(02):225-227

5 Kumar H, Das S, Rath G. An anatomical insight into the third head of biceps brachii muscle. Bratisl Lek Listy (Tlacene Vyd) 2008;109 (02):76-78

6 Donmez OB, Demiral MB, Ozsoy U, Utuk A, Savikcioglu L. Variation of the supernumerary head of the biceps brachii muscle: two case reports and literature review. International Journal of Experimental and Clinical Anatomy 2010;4:63-66

7 Balasubramanian A. Case report: supernumerary head of biceps brachii. Int J Anat Var 2010;3:214-215

8 Singh P, Tandon A, Bhatnagar R, Pokhrel R. Case report: bilateral three headed biceps brachii muscle. Medical Journal of Shree Birendra Hospital 2013;12(02):58-60

9 Govindarajan A, Vellaichamy V. Case report: unusual origin of third head of biceps brachii. Innovative Journal of Medical and Health Science 2013;8(03):156-157

10 Shashikala RL, Ashwini SJ. Case report: Third head of biceps brachii muscle a case study. Biomed Res 2011;22(03):387-389

11 Lokanadhan S, Devi SV. Unusual presentation of supernumery head of biceps brachii in South Indian Population. World Journal of Medical Sciences 2011;6(03):115-120

12 Cheema P, Singla R. Low incidence of the third head of biceps brachii in the North Indian Population. J Clin Diagn Res 2011;5 (07):1323-1326

13 El-Naggar MM, Zahir FI. Two bellies of the coracobrachialis muscle associated with a third head of the biceps brachii muscle. Clin Anat 2001;14(05):379-382 Doi: 10.1002/ca.1067

14 Nayak SR, Krishnamurthy A, Prabhu LV, Jiji PJ, Ramanathan L, Kumar S. Multiple supernumerary muscles of the arm and its clinical significance. Bratisl Lek Listy (Tlacene Vyd) 2008;109(02):74-75 